\title{
Some evidence for correlated separate activation in a simple letter-detection task
}

\author{
A. H. C. van der HEIJDEN, R. SCHREUDER, L. MARIS, and M. NEERINCX \\ University of Leiden, Leiden, The Netherlands
}

\begin{abstract}
This study investigates the separate activation versus coactivation issue for redundant targets in a simple letter-detection paradigm with latency as the dependent variable. The results of a one-response visual-search task are reported. Since, on single-target trials, only the target was presented and no accompanying noise element, no "distraction decrement" caused by irrelevant noise elements (Grice et al., 1984) was to be expected. The data obtained showed a clear redundantsignal effect. Subsequent detailed analysis of the latency data using Miller's (1982) procedure indicated that the results were consistent with a separate activation model and failed to provide convincing evidence in favor of coactivation models. A further analysis of the data indicated that, in the present study, the separate channels were negatively correlated for a range of fast RTs and positively correlated for intermediate and larger RTs. No evidence in favor of Grice et al.'s (1984) distraction-decrement hypothesis was found. The conclusions of this study are that (1) a separate activation model summarizes the essential features of information processing in this simple visual search task, and (2) no convincing evidence in favor of coactivation in visual search tasks has been reported in the literature up to now.
\end{abstract}

In this paper, we investigate the separate activation versus coactivation issue for redundant signals in a simple visual letter-detection paradigm with latency (RT) as the dependent variable. Separate activation models assume that signals (or targets) on different channels produce separate activations in each channel, any one of which increases to a level at which it can initiate a response. Coactivation models assume that signals on different channels somewhere in the system produce combined activation, and that this combined activation initiates the response (see Miller, 1982, for a good introduction).

It is clear that information about the separate activation versus coactivation issue is essential for modeling the human (visual) information processor. Overall, mean RTs do not provide this information. This is especially so because both separate activation and coactivation models can explain a "redundant signal effect," that is, the faster responding when two (or more) signals are presented simultaneously than when only one signal is presented in isolation. Separate activation models explain this effect in terms of a race between the separate activations. Given randomly varying durations for the individual processes, the average duration of the winning process in the race is smaller than the average duration of the individual processes (see, e.g., Van der Heijden, La Heij, \& Boer, 1983). Coactivation models explain this effect in terms of summed activation. Summed or combined activation

A. H. C. van der Heijden is also associated with the Zentrum für interdisziplinaire Forschung der Universität Bielefeld, Federal Republic of Germany. R. Schreuder is now at the Interfaculty Research Unit for Language and Speech, University of Nijmegen. Requests for reprints should be sent to: A. H. C. van der Heijden, University of Leiden, Hooigracht 15, 2312KM Leiden, The Netherlands. can satisfy a single criterion faster than a single activation can. Therefore, an observed redundant signal effect (or a redundancy gain) is compatible with both types of models, and a more detailed analysis of RTs is necessary for obtaining the necessary information with regard to coactivation versus separate activation.

Recently, Miller (1978, 1981, 1982) presented a simple and elegant test for obtaining the information needed. We describe this test under "Method of Analysis." Miller (1982) used the test for evaluating the results obtained in three bimodal detection tasks and two visual letter-search tasks. He concluded that the data from both types of tasks appeared inconsistent with separate activation models and favored coactivation models (see Miller, 1982, abstract). However, for the letter-search tasks there are a number of reasons to doubt this conclusion. We briefly elaborate some of these reasons.

First, the evidence in favor of coactivation that Miller (1982) finds in his letter-search tasks is rather weak. With regard to his Experiment 4, he concludes that the evidence must be regarded as somewhat tentative, since his test showed only a few, and barely significant, indications in favor of coactivation (see Miller, 1982, p. 265). A second test, using a subset of the data of his Experiment 5 , replicates nearly exactly the pattern obtained in Experiment 4 (see Miller, 1982, p. 267). A third test, using another subset of the data fails to provide any evidence at all in favor of coactivation (see Miller, 1982, p. 268).

Second, Miller's conclusion seems to be at variance with other results reported in the literature. Meijers and Eijkman (1977) used a flash-detection task with RT as the dependent variable and varied the interval between flashes on redundant-flash trials. A race model with independent parallel processing provided the better fit to the data and 
a coactivation model had to be rejected. C. W. Eriksen and colleagues (C. W. Eriksen, 1966; C. W. Eriksen \& Lappin, 1965, 1967; Collins \& C. W. Eriksen, 1967) investigated performance in a number of letter-detection tasks with identification accuracy as the dependent variable. Their main interest was in establishing whether there were enough independent sensory channels for perceiving the letters. The general conclusion from this line of research was that each occurrence of a target on redundant target trials represented an independent opportunity for the target to be perceived when the stimuli were separated by more than $1^{\circ}$, whereas sensitivity or error was positively correlated with a separation of less than $1^{\circ}$. Of course, the latter results do not rule out coactivation in speeded-response tasks after recognition of the signals in the process of activating the decision to respond (see Miller, 1982, pp. 269-270). As stated, however, Meijers and Eijkman (1977) found no evidence at all for such coactivation in their speeded-response task.

Third, Miller (1982) used two-response targetpresent/target-absent search tasks. Furthermore, in the conditions that led him to the coactivation conclusion, the single targets requiring a target-present response, were accompanied by noise elements, requiring a target-absent response on noise-only trials. In another line of research, C. W. Eriksen and his colleagues (see B. A. Eriksen \& C. W. Eriksen, 1974; C. W. Eriksen \& Schultz, 1979) have shown that such noise letters can produce pronounced effects at the response level in two-response tasks. Van der Heijden and La Heij $(1982,1983)$ obtained collaborating evidence. They showed that there were differences in RTs, as a function of number of noise elements presented simultaneously with a target, between oneresponse (i.e., go/no-go) tasks and two-response (i.e., yes-no and detection) tasks. So, it is possible that, instead of faster responding as a result of coactivation on redundant target trials, delayed responding as a result of response interference on single-target trials is the basis of Miller's (1982) evidence in favor of coactivation models in simple search tasks. In fact, in the part of Miller's study that provided no evidence in favor of coactivation, the single targets were presented without accompanying noise elements!

Recently, Grice, Canham, and Boroughs (1984) correctly noted this response competition problem in Miller's (1982) letter-detection tasks. To investigate the separate activation versus coactivation issue, these authors used a choice reaction task with two target letters, one mapped on each of two responses. One of these two letters was presented either on two channels or singly, accompanied by an irrelevant letter. It was assumed that, with this procedure, the irrelevant letter would evoke no response and the response competition problem would be avoided. In their letter-detection tasks (Experiments 3, 4, and 5), application of Miller's test indicated some evidence in favor of coactivation, but appreciably less than that found by Miller (1982, p. 267). Thus, response competition was indeed a factor of importance in Miller's (1982) experi- ments. The main conclusion of Grice et al, was that coactivation, and not separate activation, underlay subjects' performance.

Grice et al, then started a search for the form of coactivation or for the combination rule. In the combination rule they proposed, each component on redundant target trials contributes about one-half of its strength (strength is a simple transformation of the obtained variable, response proportion, and time). But there is also an essential additive component in the combination rule, and that component apparently produces the redundant signal effect. The problem lies in how to interpret this constant. Grice et al.'s (1984) interpretation was very simple: "Our findings suggest ... that the strength of each component may be a little greater on two-target trials. . . . [T] he strength of one component may be reduced if the other position or level is occupied by a distractor rather than by another target. This reduction might be called a distraction decrement" (p. 461).

This interpretation, however, completely undermines the main conclusion drawn from Miller's test, that is, the conclusion that it is coactivation and not separate activation that underlies subjects' performance. If there is, indeed, a distraction decrement on single-target trials, no coactivation assumption is needed to explain faster responding on redundant-target trials. Also a separate activation model with this distraction decrement assumption added is perfectly consistent with such a result. (What Grice et al. suggest in their interpretation is that the difference between single- and double-target trials may be due, at least in part, to interference that accompanies a single target but is absent when two targets are present. But this is exactly the formulation Grice et al., p. 452, use to describe the problem response competition creates for interpreting Miller's, 1982, research.) If there is a distraction decrement on single-target trials, application of Miller's test only leads to results that are very difficult to interpret with regard to the separate activation versus coactivation issue. Only if it can be assumed that there is no such distraction decrement (nor a redundant target increment; see Grice et al., 1984, p. 462) can Miller's test provide the wanted information.

In this paper, we report the results of a visual-search experiment designed to investigate the separate activation versus coactivation issue. On single-target trials, only a target was presented and no irrelevant element, so no distraction decrement was expected. To avoid responsecompetition problems, a one-response, go/no-go task was used. Such a task has additional advantages in that it is not much affected by practice and yields a very low number of error responses (see Van der Heijden \& La Heij, 1982,1983 ). To prevent useful eye movements during stimulus exposure, an exposure duration of $150 \mathrm{msec}$ was used. In Miller's (1982) and Grice et al.'s (1984) experiments, the stimuli remained on until the response was made. While this difference in exposure times might complicate comparisons between the experiments, short exposure times are to be preferred if central visual infor- 
mation processing, unaided by peripheral adjustments, is the topic of investigation.

\section{METHOD OF ANALYSIS}

Miller (1982) proposed a general test of the assumption of separate activation. Separate activation models have as a basic assumption that with redundant targets, detection time is determined by the fastest of the individual target detection processes. For two simultaneously presented targets (T1 and T2), this implies that, for all values of $t$,

$$
\begin{aligned}
& \mathrm{P}(\mathrm{RT}<\mathrm{t} \mid \mathrm{T} 1 \text { and } \mathrm{T} 2) \\
&= \mathrm{P}(\mathrm{RT}<\mathrm{t} \mid \mathrm{T} 1)+\mathrm{P}(\mathrm{RT}<\mathrm{t} \mid \mathrm{T} 2) \\
& \quad-\mathrm{P}[(\mathrm{RT}<\mathrm{t} \mid \mathrm{T} 1) \text { and }(\mathrm{RT}<\mathrm{t} \mid \mathrm{T} 2)] .
\end{aligned}
$$

The left side of the equation corresponds to the cumulative probability density function (CDF) on redundant target trials. The first two terms on the right correspond to the CDFs on the two types of single-target trials, but only if it can be assumed that target processing on single-target trials is equivalent to target processing on redundant target trials. If this assumption holds, estimates for these terms can be derived from experimental data. In general, the last term reflects the correlation between the two simultaneous target detection processes, or channels, on redundant target trials.

Miller's test uses the fact that the last term in the equation equals or is greater than 0 , that is, that

$$
\mathrm{P}[(\mathrm{RT}<\mathrm{t} \mid \mathrm{T} 1) \text { and }(\mathrm{RT}<\mathrm{t} \mid \mathrm{T} 2)] \geq 0 .
$$

It follows, therefore, that with separate activation, for all values of $t$,

$\mathrm{P}(\mathrm{RT}<\mathrm{t} \mid \mathrm{T} 1$ and $\mathrm{T} 2) \leq \mathrm{P}(\mathrm{RT}<\mathrm{t} \mid \mathrm{T} 1)+\mathrm{P}(\mathrm{RT}<\mathrm{t} \mid \mathrm{T} 2)$.

Thus, all separate activation models have to be rejected if this inequality is violated, that is, if the (estimated) probability of occurrence of RTs smaller than some value $t$ in the redundant target condition exceeds the sum of the (estimated) probabilities in the two single-target conditions. Coactivation models allowing pooling of activation resulting from $\mathrm{T} 1$ and $\mathrm{T} 2$ are consistent with violation of Inequality 2 , because, with pooling of activation, the fastest responses to redundant signals can be faster than the fastest response to each target alone (see Miller, 1982, pp. 253-254.).

It is important to note that Inequality 2 can be violated only for relatively small values of $t$. With increasing values of $t$, the term at the left approaches 1.00 and the sum of the two terms at the right approaches 2.00 . So, for the larger values of $t$, Inequality 2 surely holds. This, however, is not problematic for the test, because coactivation models especially predict violations of Inequality 2 for the smaller values of $t$; with coactivation, combination of activation allows satisfying a single criterion before either source has provided sufficient activation alone (see Miller, 1982, p. 254).

Inequality 2 can be used only to assess whether observed data are consistent with the separate activation assumption. If the data are consistent with the assumption, it is worthwhile to further investigate what type of separate activation model the data are consistent with. Of importance here is the distinction between dependent(positively or negatively correlated)-channels and independentchannels-separate-activation models. Predictions for the independent-channels-separate-activation model can be generated with

$$
\begin{aligned}
& \mathrm{P}(\mathrm{RT}<\mathrm{t} \mid \mathrm{T} 1 \text { and } \mathrm{T} 2) \\
&= \mathrm{P}(\mathrm{RT}<\mathrm{t} \mid \mathrm{T} 1)+\mathrm{P}(\mathrm{RT}<\mathrm{t} \mid \mathrm{T} 2) \\
& \quad-\mathrm{P}(\mathrm{RT}<\mathrm{t} \mid \mathrm{T} 1) \times \mathrm{P}(\mathrm{RT}<\mathrm{t} \mid \mathrm{T} 2)
\end{aligned}
$$

(see Meijers \& Eijkman, 1977; Miller, 1982). A comparison between these predictions and the data obtained in the redundant target condition can indicate what type of separate activation model is in accord with the data. This can also be indicated, as demonstrated by Grice et al . (1984), by computing a tetrachoric correlation.

\section{METHOD}

\section{Apparatus}

The stimuli were presented on a fast display screen (Vector General Graphics Display, V11) equipped with P4-phosphor. The display was connected with a PDP-11/34 minicomputer. The program used took care of the presentation of the stimuli in random order, the registration of the reaction times (RTs), and the classification of the responses (correct responses vs. errors per type of trial). RTs were recorded accurate to $1 \mathrm{msec}$. A footbar was provided for the subjects for initiating stimulus exposure themselves.

\section{Stimuli}

The stimulus elements used were continuous-line "white" Roman capitals on a dark background, generated by the Vector General Display software. Only the letters E (designated as the target letter for all subjects) and $O$ (the noise letter) were used. The letters were presented at the left and/or at the right of a central fixation point (a dim asterisk). Their center was located $.69^{\circ}$ from the fixation point. The size of the letters was approximately $.27^{\circ} \times .18^{\circ}$ in visual angle.

In each block of 30 experimental trials, six types of trials occurred equally often: (1) $\mathrm{E}$ in the left position but not in the right (five trials), (2) $\mathrm{E}$ in the right position but not in the left (five trials), (3) an $E$ in both positions (five trials), (4) $O$ in the left position but not in the right (five trials), (5) $\mathrm{O}$ in the right position but not in the left (five trials), and (6) an $\mathrm{O}$ in both positions (five trials).

\section{Subjects}

Twenty-four students at the University of Leiden served as paid subjects. They all had normal or corrected-to-normal vision. They had never participated in a similar experiment before. 


\section{Procedure}

The subjects were run individually in a moderately illuminated room. The subject was instructed to look at the display until he/she clearly saw the fixation point. $\mathrm{He} / \mathrm{sh}$ was to press the footbar with the right foot $700 \mathrm{msec}$ after which the stimulus appeared. Exposure duration was $150 \mathrm{msec}$.

One response button was used. The subject was instructed to keep his/her right index finger above this button, to respond when one or two Es appeared, and to refrain from responding when one or more Os appeared. They were asked to respond as fast as possible while maintaining accuracy. Only reactions within an interval of $1,500 \mathrm{msec}$ after stimulus exposure were considered as targetpresent responses.

Each subject served on 2 consecutive days. On each day, one series of 24 and five series of 34 trials were presented. The first series on each day and the first 4 trials of the following series were randomly chosen practice trials. Only the RTs of the remaining Epresent trials were used in the analysis. So, per subject, there were 2 (days) $\times 5$ (series) $\times 5$ (trials) $=50$ trials for $E$ left, $E$ right, and for EE stimuli.

\section{RESULTS AND DISCUSSION}

Average RTs were computed separately for each subject and per type of target-present trials. The mean RTs across subjects for E-left, E-right, and E-E trials were 340,345 , and $325 \mathrm{msec}$, respectively. An analysis of variance showed that the difference between these means was highly significant $[F(2,46)=29.97, p<.001]$. Subsequent pairwise comparisons, using Tukey's HSD test, revealed significant differences between $E-E$ trials and both types of single $E$ trials $(p<.01)$. The difference between the two types of single $E$ trials was far from significant. In summary, the data show the usual and expected redundant signal effect.

It is possible, however, that this redundant signal effect is only an artifact, resulting from averaging across subjects per single-target condition. Suppose that some subjects processed Es at the right faster than Es at the left and other subjects processed Es at the left faster than they did Es at the right. This way neither the E-left mean nor the E-right mean estimates the average across subjects of their best, that is, fastest, performance in the single-target conditions (see Biederman \& Checkosky, 1970; Nickerson, 1972, and Van der Heijden et al., 1983, for further discussions of this artifact). To show that the redundant signal effect is real, we computed the average across subjects of the faster of the two single signal conditions. The value obtained was $336 \mathrm{msec}$. This value is still significantly greater than the $325 \mathrm{msec}$ found for the redundant target condition $[t(23)=5.52, \mathrm{p}<.001]$. At this point, it is worthwhile to note that this value is smaller than the values for E-left ( $340 \mathrm{msec}$ ) and E-right ( $345 \mathrm{msec})$, so part of the original redundant-signal effect was only an averaging artifact (of the 24 subjects, 15 were fastest in the E-left and 9 were fastest in the E-right condition).

It is likely that part of Grice et al.'s (1984) redundanttarget effects also results from this averaging artifact (see their pages 457 and 459). It is, furthermore, worthwhile to note that the redundant-target effect found in the present experiment was obtained with single-target stimuli without accompanying noise elements. This is a first indication that Grice et al.'s (1984) distraction decrement hypothesis, that is, the suggestion that "the "redundant signals effect' in this situation is not produced by the additional information from a second target but by the absence of a distractor' (p. 462), is incorrect. (Notice how Grice et al.'s formulation in fact denies the coactivation hypothesis!)

In Figure 1, the average CDFs for the E-left (the line labeled 2), E-right (3), and E-E (1) trials are graphically represented. The first step in the procedure to obtain these CDFs is the organizing in ascending order of the RTs in each condition for each subject. For each subject in each condition, 50 RTs were obtained. Therefore, each ordered row of RTs provides an estimate of the 1st, 3rd, 5th ... 97th and 99th percentile of the CDF for one subject and condition. The average CDFs are obtained by averaging, per condition across subjects, the RTs at each percentile. This method of obtaining an average RT distribution for a group of subjects is described in Ratcliff (1979). The prime advantage of this method is that the average CDF retains the shape information of the individual CDFs, that is, properties derived from the group reaction time distribution are the same as average properties derived from the data of individual subjects (see Ratcliff, 1979).

We evaluated Inequality 2 by following the method introduced by Miller (1982). The method starts with the CDFs per subject and single-target condition. Next, for each subject, the right-hand sum is determined by summing for each RT the (interpolated) percentiles of his/her two single-signal CDFs and selecting the RTs where the sum equals $1,3,5 \ldots 97,99$. Last, the average righthand sum of Inequality 2 is obtained by averaging, across subjects, the RTs at each percentile. In Table 1, the column labeled A gives the RTs so obtained for a num-

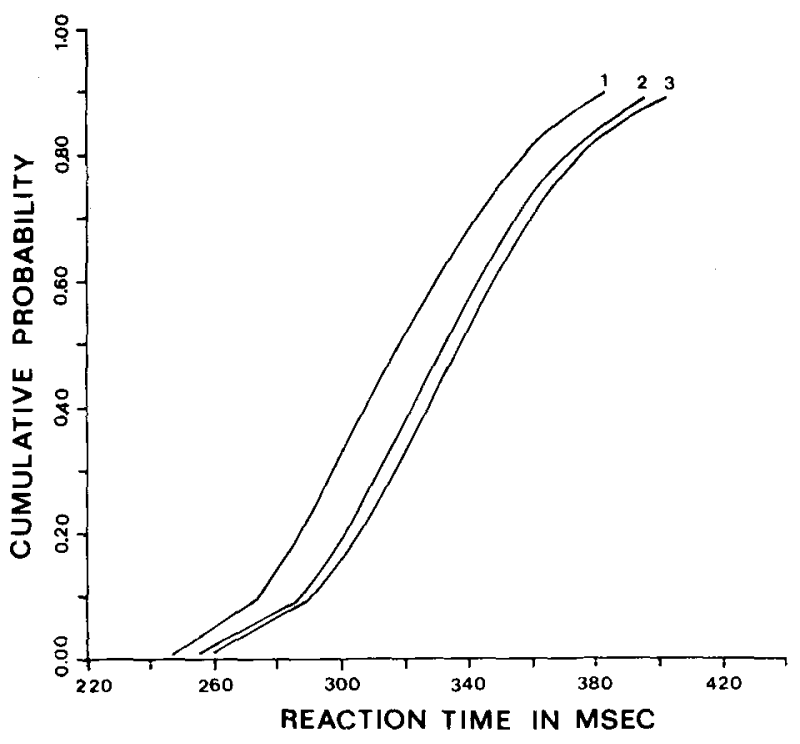

Figure 1. Cumulative probability density functions (CDFs) for $E$ left (2), E-right (3), and E-E (1) conditions. 
Table 1

Initial and Some Higher Values of Single-Signal CDFs (Er and El), Redundant Signal CDF (O), and Two Types of Combinations of Single-Signal CDFs (A and B)

\begin{tabular}{|c|c|c|c|c|c|c|c|c|}
\hline$\underline{\mathbf{P}}$ & Er & El & 0 & A & O-A & B & O-B & $\mathrm{R}$ \\
\hline 1 & 260 & 256 & 248 & 251 & -3 & 251 & -3 & \\
\hline 3 & 275 & 268 & 260 & 260 & 0 & 260 & 0 & \\
\hline 5 & 283 & 274 & 266 & 264 & 2 & 264 & +2 & \\
\hline 7 & 286 & 280 & 270 & 269 & 1 & 269 & +1 & \\
\hline 9 & 290 & 286 & 273 & 274 & -1 & 274 & -1 & \\
\hline 11 & 294 & 289 & 277 & 278 & -1 & 278 & -1 & \\
\hline 13 & 297 & 292 & 279 & 281 & -2 & 282 & -3 & \\
\hline 15 & 300 & 295 & 282 & 283 & -1 & 284 & -2 & \\
\hline 17 & 302 & 298 & 284 & 286 & -2 & 286 & -2 & \\
\hline 19 & 306 & 300 & 287 & 288 & -1 & 288 & -1 & \\
\hline 21 & 309 & 303 & 289 & 290 & -1 & 291 & -2 & \\
\hline 23 & 312 & 306 & 292 & 292 & 0 & 293 & -1 & -1.00 \\
\hline 25 & 313 & 308 & 294 & 294 & 0 & 295 & -1 & -.61 \\
\hline 27 & 315 & 310 & 296 & 295 & 1 & 297 & -1 & -.31 \\
\hline 29 & 317 & 312 & 298 & 297 & 1 & 298 & 0 & -.17 \\
\hline 31 & 319 & 314 & 301 & 298 & 3 & 300 & +1 & .25 \\
\hline 33 & 321 & 316 & 302 & 299 & 3 & 301 & +1 & .14 \\
\hline 35 & 323 & 317 & 304 & 301 & 3 & 303 & +1 & .11 \\
\hline 37 & 326 & 320 & 306 & 302 & 4 & 304 & +2 & .09 \\
\hline 39 & 327 & 322 & 308 & 303 & 5 & 306 & +2 & .15 \\
\hline 41 & 329 & 324 & 310 & 304 & 6 & 308 & +2 & .20 \\
\hline 43 & 330 & 326 & 312 & 306 & 6 & 309 & +3 & .23 \\
\hline 45 & 331 & 329 & 314 & 307 & 7 & 311 & +3 & .34 \\
\hline 55 & 342 & 339 & 326 & 313 & 13 & 318 & +8 & .58 \\
\hline 65 & 355 & 350 & 338 & 318 & 20 & 326 & +12 & .72 \\
\hline 75 & 368 & 363 & 351 & 323 & 28 & 335 & +16 & .76 \\
\hline 85 & 388 & 384 & 370 & 328 & 42 & 347 & +23 & .85 \\
\hline 95 & 509 & 438 & 402 & 333 & 69 & 370 & +32 & .76 \\
\hline
\end{tabular}

Note-Column $P$ gives the corresponding percentile values and Column $R$ the correlations between channels on redundant target trials. See text for further explanation.

ber of, for this discussion, relevant percentiles. The column labeled $O$ gives the obtained average redundant signal CDF for the same percentiles. These values are estimates of the term at the left in Inequality 2 . The column labeled $(\mathrm{O}-\mathrm{A})$ presents the differences between the values in the columns labeled $\mathrm{O}$ and $\mathrm{A}$. This column shows that there is no reason to assume that the inequality is violated for the smallest percentile values. (A t test showed that the initial negative value, -3 , was far from significant.) This outcome is essential because coactivation models predict violations, especially for the smallest percentile values; with coactivation, only the fastest responses to redundant signals could be faster than the fastest responses to either signal alone (see Miller, 1982, pp. 253-254). Because, for small values of $P$, the last term in Equation 1 equals or is very close to zero, the results obtained with the smaller percentile values are in good agreement with Equation 1, and therefore consistent with all separate activation models considered in this study.

The column labeled (O-A), however, also shows that there is a range of seven percentile values, from the 9th to the 21 st, at which Inequality 2 is slightly, but consistently, violated. None of these violations even approached significance. Inspection of the individual distributions showed that $15,12,11,12,12,12$, and 11 of the 24 subjects showed no violations of Inequality 2 at the 9 th, 11 th, ..., and 21 st percentiles. These results, together with the fact that no consistent violations are found for the first four percentile values, make it very unlikely that the negative values in Column $\mathrm{O}-\mathrm{A}$ have to be interpreted as evidence in favor of coactivation. Sequence effects (see Miller, 1982, p. 271) or fast guesses can account for this result too.

Taken together, the results fail to falsify the separate activation hypothesis. (At this point, it is worthwhile to remember that Miller, 1982, too, found no evidence in favor of coactivation in the part of his study in which single targets were presented without noise elements.) Given this state of affairs, it is worthwhile to further investigate what type of separate activation model-independent channels versus (positively or negatively) correlated channels-is consistent with the results obtained.

As stated under Method of Analysis, the positive values for the higher percentiles in Column O-A provide no information because Inequality 2 entails such a result; with increasing values of $t$, the term at the left approaches 1.00 and the sum at the right approaches 2.00. This is not the case, however, for Equation 3, representing the independent-channels separate-activation model. Therefore, this model can be used to predict expected RTs on redundant-target trials and deviations between these predicted and the observed RTs can inform us about the most likely type of separate activation model.

Per subject, the expected independent-channels, redundant-signal CDF (i.e., the right-hand side of Equation 3) was determined by first summing, for each RT of his/her single-signal CDFs, the (interpolated) percentiles and subtracting the product of the percentiles (percentiles were expressed in values between 0 and 1). Then the RTs for the 1 st, 3rd, ... percentile were selected. The average expected redundant-signal CDF was obtained by averaging across subjects the RTs per percentile value. The column labeled $B$ in Table 2 presents this CDF. The column labeled $\mathrm{O}-\mathrm{B}$ presents the deviations between the average expected redundant-signal CDF and the observed redundant-signal CDF.

Column O-B shows that the obtained redundant-target RTs deviate systematically from the RTs predicted with an independent-channels separate-activation model. From the 9th up to the 27th percentile, observed RTs are somewhat smaller than predicted RTs. Starting with the 31st percentile, the differences are positive and increase with increasing percentile values, that is, the observed RTs are larger than the predicted RTs. As stated, the differences listed in Column O-B indicate real deviations from the independent-channels model and are not an artifact of the way the test is used in this case. The problem lies in how to interpret the sign $(+, 0,-)$ of these deviations.

One way to determine the direction of the correlation starts with comparing probabilities for identical R'Ts. For 
example, in Column $\mathrm{B}$, we find that $\mathrm{P}(\mathrm{RT}<306)=.39$. Because this is the prediction for independent channels, we can write

$$
\begin{aligned}
& \mathrm{P}(\mathrm{RT} 1<306)+\mathrm{P}(\mathrm{RT} 2<306) \\
& -[\mathrm{P}(\mathrm{RT} 1<306) \times \mathrm{P}(\mathrm{RT} 2<306)]=.39 .
\end{aligned}
$$

In the column labeled $\mathrm{O}$, we find that the observed value equals $\mathrm{P}(\mathrm{RT}<306)=.37$. Given separate activation, we can write

$$
\begin{aligned}
& \mathrm{P}(\mathrm{RT} 1<306)+\mathrm{P}(\mathrm{RT} 2<306) \\
& \quad-\alpha[\mathrm{P}(\mathrm{RT} 1<306) \times \mathrm{P}(\mathrm{RT} 2<306)]=.37 .
\end{aligned}
$$

From (3a) and (3b), it follows that

$$
\begin{aligned}
& \alpha[\mathrm{P}(\mathrm{RT} 1<306) \times \mathrm{P}(\mathrm{RT} 2<306)] \\
&>[\mathrm{P}(\mathrm{RT} 1<306) \times \mathrm{P}(\mathrm{RT} 2<306)]
\end{aligned}
$$

or that $\alpha>1$, and that indicates a positive correlation between channels on redundant target trials (an $\alpha<1$ indicates a negative correlation, and $\alpha=1$ independence; see also Grice et al., 1984, p. 452). With this procedure, it is easy to show that all plus values in Column O-B indicate a positive correlation, all minus values a negative correlation, and zeros, independence. Thus, this analysis leads to the conclusion that after the first 4 percentile points there is a range of 10 percentile points where a separate activation model with negatively correlated channels provides the best fit. Starting with the 31 st percentile point, a separate activation model with positively correlated channels has to be postulated to account for the data.

Recently, Grice et al. (1984, p. 458) introduced an elegant method to obtain quantitative theoretical estimates of the correlation between channels on redundant target trials. Fourfold tables, from which a tetrachoric correlation may be computed, are constructed. The proportions of responding and not responding to the two types of single targets provide estimates of the marginals of the table. The probability of not responding on redundant target trials fills one cell of the matrix. This determines the entire table (the table has only one degree of freedom).

From the average distributions, we computed cosinepi estimates of tetrachoric correlation at the percentile values of the redundant target distribution. Column $R$ in Table 1 presents these values. (For the smaller percentile values, the fourfold tables constructed in the way described contain small negative proportions in the bothsmaller-than-t entry, and computation of the cosine-pi estimate is impossible.) The negative values in Column $\mathbf{R}$ again indicate a range of values, with which a separate activation model with negatively correlated channels is in accord. Starting with the 31 st percentile, a positive correlation between separate channels has to be postulated.
It seems difficult to explain this rather unexpected pattern of results. However, assume that on a subset of trials (some) subjects move their direction of gaze (close) to the position where they expect a target to occur. If a target appears at that position, it is projected on the position of the retina with highest visual acuity and a fast RT is to be expected. If a target appears in the other position, it is projected on a part of the retina that has appreciably lower visual acuity, and a much larger RT is to be expected. On single-target trials, on the average, subjects will be about $50 \%$ correct in predicting the position of the impending target. On redundant target trials, however, $100 \%$ of these predictions are correct. (Given the design of our experiment, across conditions subjects would be about $66 \%$ correct in predicting the target position; therefore, the proposed strategy would not be an unreasonable one for the subjects to adopt.) This "fixational bias hypothesis" readily explains why it is only with faster RTs that the channels are negatively correlated on redundant target trials; given the fixational bias, on redundant target trials, the fixated target is processed extra fast but the nonfixated target is processed extra slowly relative to target processing with correct fixation. It is likely, however, that on the majority of trials subjects fixate correctly at the fixation point. Then intermediate RTs for both positions are to be expected. It appears that these intermediate RTs are positively correlated (see also Grice et al., 1984, Experiments 4 and 5). Below, we briefly return to these positive correlations. In summary, the present data are in accord with a separate activation model with (1) positively correlated channels with correct fixations (intermediate and large redundant target RTs) and (2) negatively correlated channels as a result of fixational biases (fast RTs on redundant target trials).

It is, however, not necessary to invoke fixational biases to explain our results in terms of a separate activation model. C. W. Eriksen and Hoffman (1974) demonstrated clear facilitating effects of foreknowledge of position in a single-letter-recognition task with latency as the dependent variable. Posner, Nissen, and Ogden (1978) and Posner, Snyder, and Davidson (1980) demonstrated improvements in latencies ("benefits") to luminance increments in an expected position and increases of latencies ("costs") to luminance increments in unexpected positions. For these experiments, eye movements can be excluded as an explanation. So, internal "movements of attention" can also explain the pattern of results obtained; the data are in accord with a separate activation model with (1) positively correlated channels with correctly divided attention (intermediate and large redundant target RTs), and (2) "costs" and "benefits" (i.e., a negative correlation) as a result of attentional biases (fast RTs on redundant target trials). At present, it is not clear which of the two hypotheses - the fixational bias hypothesis or the attentional bias hypothesis-has to be favored.

Taken together, the results of our experiment are in good agreement with a separate activation model, if a fixational or attentional bias assumption is added. This, 
however, does not rule out that the results are also consistent with one or another coactivation model. Fortunately, Grice et al. (1984) proposed an explicit combination or summation rule that they regard as a law of considerable generality. It is therefore possible to investigate whether our results make sense in terms of this general summation rule. In addition to a parameter indicating the contribution of each component on redundant target trials, the rule contains an additive constant, interpreted by Grice et al. as measuring a distraction decrement (see also our introduction). For present purposes, this additive constant is essential. For Grice et al.'s experiments, this constant makes sense because they presented single targets together with a distractor. In our experiment, however, there were no distractors on single-target trials. So, if Grice et al.'s summation rule really applied to the data of our experiment, no distraction decrement would be expected (i.e., an additive constant of 0 would be expected).

To see how the combination rule deals with the data presented here, we followed Grice et al.'s (1984) procedure, with the only exception being that we did not correct the cumulative distribution functions (the reader is referred to Grice et al., 1984, for the details).

First, functions of the form

$$
A(t)=a-m e^{-k t},
$$

in which $\mathrm{A}$ is a transformation of the obtained variable response proportion, $t$ is time, and $a, m$, and $k$ are constants, were determined by curve-fitting procedures for the two types of single-target trials and for the redundanttarget trials. Table 2 presents the parameters. In the upper three rows are the parameters of the functions fitted over 19 percentile values (i.e., the 5 th, 10 th, . . , 95th percentile). For the lower three rows, the 95 th percentile was deleted. The column labeled "Sum of Squared Residuals" shows that the fit is appreciably better when the discrepant tailpoints at the 95th percentile are not included. The latter set of functions was used in determining the parameters of the combination rule.

As for Grice et al.'s (1984) data, for our experiment the function for targets in both positions is a nearly perfect linear function of the sum of the single-target functions plus an additive constant; that is,

Table 2

Estimated Parameters of "Strength" Functions for E Right, E Left, and E-E (Equation 4)

\begin{tabular}{lcccc}
\hline & $\mathrm{a}$ & $\mathrm{m}$ & $\mathrm{k}$ & $\begin{array}{c}\text { Sum of } \\
\text { Squared } \\
\text { Residuals }\end{array}$ \\
\hline E Right & 2.0606 & -102.2805 & -.01164 & .12399 \\
E Left & 3.2070 & -36.5691 & -.00731 & .02453 \\
E-E & 3.7667 & -30.2786 & -.00651 & .02256 \\
E Right & 3.9896 & -32.8402 & -.00625 & .00957 \\
E Left & 4.1313 & -28.2795 & -.00577 & .00736 \\
E-E & 3.1132 & -37.4506 & -.00779 & .00805 \\
\hline
\end{tabular}

Note-Upper set is fitted over 19 percentiles $(5$ th, 10th, . . , 95th) lower set is fitted over 18 percentiles (95th percentile deleted).
A Both (t)

$$
=.448[\mathrm{~A} \text { left }(\mathrm{t})+\mathrm{A} \text { right }(\mathrm{t})]+.342\left(\mathrm{R}^{2}=.999\right) \text {. }
$$

But, although this relation describes the data perfectly, how to interpret it is far from clear. We find an additive constant, interpreted by Grice et al. as measuring a distraction decrement of .342 , but no distractors were presented on single-target trials! Furthermore, this value is well within the range of values reported by Grice et al. (1984) for experiments in which single targets were accompanied by noise elements (.313-.455). Of course, it can be argued that the present experiment is not directly relevant to Grice et al.'s distraction hypothesis, because the intercept might have been higher if distractors had been presented. But the issue is not that the intercept might have been higher, but that it is not lower. A "distraction" decrement without distractors that is as large as it is with distractors makes it doubtful that the intercept really reflects a distraction decrement in our experiment. ${ }^{1}$ Our conclusion is that Grice et al.'s summation rule, in the way they interpret it, does not apply to our data. ${ }^{2}$

The above analysis strongly suggests that Grice et al.'s summation rule as they interpret it is not a correct alternative explanation for the results obtained in our experiment. Let us now take a brief look at the visual search data that led Grice et al. to reject separate activation theories and suggested some form of summation principle in terms of strength. Grice et al.'s reason for rejecting separate activation theories is that their data violate Miller's inequality (see Grice et al.'s pp. 458 and 460). In this respect, one further feature of their data is of importance.

It appears that the amount of evidence against the separate activation theories that Grice et al. (1984) find increases with increasing distance of the elements from the fixation point. With a distance of $.25^{\circ}$, they found no violations of Inequality 2 (their Experiment 4); with a distance of $1.5^{\circ}$, they found nonsignificant violations at the 5th and 10th percentiles (Experiment 5); and with a distance of $3^{\circ}$, they found a significant violation at the 5 th percentile and nonsignificant violations at the 10 th, 15th, and 20th percentiles (Experiment 3). It is far from clear how a coactivation model can cope with this pattern of results if no further assumptions are added. In fact, Miller (1982, p. 263) predicts the opposite pattern, and even obtains data that is consistent with that hypothesis, though not statistically significant (p. 265).

It is not too difficult to see what assumption has to be added to understand this pattern of results. Grice et al. (1984) attempted to avoid the response competition problems inherent in Miller's (1982) yes-no task by using a two-alternative forced-choice task with $\mathrm{S}$ and $\mathrm{H}$ as targets and $\mathrm{Y}$ as irrelevant noise letter. But C. W. Eriksen and his associates (see C. W. Eriksen \& Schulz, 1979; C. W. Eriksen, O'Hara, \& B. A. Eriksen, 1982) have demonstrated that response competition is a much more pervasive effect than it previously had been assumed. The dissimilar noise letter might indeed be neutral in that it does not prime either response if it is projected onto a 
high-acuity position of the retina. It is far from clear, however, whether it is still truly neutral if projected onto positions of lesser acuity. Then, and according to Eriksen's continuous flow model especially early in the growth of the percept, the noise letter might prime the already preprimed alternative response. If the response evocation threshold for the target is reached relatively fast, execution of the repsonse will be delayed because of the activation of the competing response. So response competition effects are also to be expected in forced-choice tasks, especially for the faster responses and when the elements are presented on retinal positions of lesser acuity (see especially C. W. Eriksen et al., 1982). This response competition assumption makes clear why Grice et al. (1984) found violations of Miller's inequality and why they found more violations with increasing separation between letters. Taken together, it seems that there was no real reason for rejecting separate activation theories for the visual search tasks to start with; the results did not really suggest some form of summation principle in terms of strength.

Our first conclusion is that the research up to now has failed to provide convincing evidence in favor of coactivation in visual search tasks. As already stated, Miller's (1982) research is flawed because the paradigm he used introduced response competition problems (see also Grice et al., 1984, p. 452). In the earlier paragraphs, we argued that Grice et al.'s experiments might have been biased in the same way, despite the explicit efforts to avoid response competition. So what remains as evidence in favor for coactivation is the precise way in which, according to the summation rule, the stimuli combine in strength (see Grice et al., 1984). Because it is completely unknown how this rule will fit given separate activation, however, this cannot be taken as real evidence in favor of coactivation.

Our second conclusion is that the results of the search task here reported are (1) consistent with, and (2) provide evidence for, correlated separate channels. The first part of the conclusion follows from the data and the analysis of the data presented. The second part of the conclusion is based on the following considerations and data. Separate activation is adaptive because actions are initiated and guided by single-stimulus events. (If there are more apples, it is more appropriate to reach for each of them in turn than to try to grasp them all at the same time.) Coactivation might be adaptive because, by the pooling of activation, a response can be initiated faster, that is, before either signal has provided enough activation to produce a response by itself. (Is it better to start fleeing earlier when there are two lions instead of one?) So far, just this advantage of coactivation has not been convincingly demonstrated. In fact, it appears-and this becomes readily apparent if we shift our attention from the very low to the intermediate and higher percentile values - that the opposite result is found. Responses to redundant targets are generally slower than expected on the basis of an independent-channels separate-activation model (see the values in the Column O-B in Table 1; see also Grice et al., 1984). The data provide no evidence at all that the visual information processing system sacrifices precision to win time. Rather it sacrifices time to gain precision.

Our second conclusion (that the results of the search task reported here provide evidence for correlated separate channels) is seemingly at variance with Meijers and Eijkman's (1977) conclusion that a race model with independent channels provides the better fit to their flash-detection data. However, because of the difficulties inherent in constructing a sensible model with partial dependence, that is, $|\mathrm{R}|<1$, they tested only the extreme assumption of stochastically dependent processes (see Meijers \& Eijkman, 1977, p. 43). So, it cannot be ruled out that a model with moderately correlated channels provided an even better fit to their data than the independent-channels model.

The data presented in this study and in Grice et al. (1984) offer, with latency data, some support for the conclusion that C. W. Eriksen and his colleagues reached on the basis of their letter-detection research with identification accuracy as the dependent variable. As stated in the introduction, their general conclusion was that an independent-channels model provides the better fit when the stimuli on redundant target trials are separated by more than $1^{\circ}$, whereas sensitivity or error is positively correlated when the stimuli are separated less than $1^{\circ}$. With a separation of $.5^{\circ}$, Grice et al. (1984, Experiment 4) obtained a mean correlation of .76; in our study, with a separation of $1.2^{\circ}$ the mean of the positive correlations was appreciably lower (see Table 1); with a distance of $3^{\circ}$ (see Grice et al., 1984, Experiment 5), the positive correlations at the higher percentile values were again lower (unfortunately, Grice et al. do not report correlations for their $6^{\circ}$-distance experiment). So it seems that the positive correlations decrease with increasing distance between the stimuli, albeit this applies to distances longer than those reported by Eriksen and his colleagues. Why positive correlations are found over distances that are larger in long stimulus-exposure latency experiments than in short stimulus-exposure accuracy experiments is a matter for further research. And, of course, more research is also needed to settle the important separate activation versus coactivation issue. Our contribution is only an attempt to keep the issue open.

\section{REFERENCES}

Biederman, I. , \& Checkosky, S. F. (1970). Processing redundant information, Journal of Experimental Psychology, 83, 486-490.

Collins, J. F., \& Eriksen, C. W. (1967). The perception of multiple simultaneously presented forms as a function of foveal spacing. Perception \& Psychophysics, $2,369-373$.

ERIKSEN, B. A., \& ERIKSEN, C. W. (1974). Effects of noise letters upon the identification of a target letter in a nonsearch task. Perception \& Psychophysics, 16, 143-149.

ERIKSEN, C. W. (1966). Independence of successive inputs and uncorrelated error in visual form perception. Journal of Experimental Psychology, 72, 26-35.

Eriksen, C. W., \& Hoffman, J. E. (1974). Selective attention: Noise suppression or signal enhancement? Bulletin of the Psychonomic Society, 4, 587-589.

ERIKSEN, C. W., \& LAPPIN, J. S. (1965). Internal perceptual system 
noise and redundancy in simultaneous inputs in form identification. Psychonomic Science, 2, 351-352.

ERIKSEN, C. W., \& LAPPIN, J. S. (1967). Independence in the perception of simultaneously presented forms at brief durations. Journal of Experimental Psychology, 73, 468-472.

Eriksen, C. W., O'Hara, W. P., \& ErIKSEN, B. (1982). Response competition effects in same-different judgments. Perception \& Psychophysics, 32, 261-270.

ERIKSEN, C. W., \& Schultz, D. W. (1979). Information processing in visual search: A continuous flow conception and experimental results. Perception \& Psychophysics, 25, 249-263.

Grice, G. R., Canham, L., \& Boroughs, J. M. (1984). Combination rule for redundant information in reaction time tasks with divided attention. Perception \& Psychophysics, 35, 451-463.

Mejers, L. M. M., \& Eukman, E. G. J. (1977). Distributions of simple RT with single and double stimuli. Perception \& Psychophysics, 22, 41-48.

Miller, J. (1978). Multidimensional same-different judgments: Evidence against independent comparisons of dimensions. Journal of $E x$ perimental Psychology: Human Perception and Performance, 4, 411-422.

MILLER, J. (1981). Global precedence in attention and decision. Journal of Experimental Psychology: Human Perception and Performance, 9. 1161-1174.

Miller, J. (1982). Divided attention: Evidence for coactivation with redundant signals. Cognitive Psychology, 14, 247-279.

NiCKERSON, R. S. (1972). Binary classification reaction time: A review of some studies of human information processing capabilities. Psychonomic Monograph Supplements, 4(Whole No. 00), 275-318.

PosNer, M. I., Nissen, M. J., \& OGDEN, W. C. (1978). Attended and unattended processing modes: The role of set for spatial location. In H. L. Pick \& I. J. Saltzman (Eds.), Modes of perceiving and processing information. Hillsdale, NJ: Erlbaum.

Posner, M. I., SNyder, C. R. R., \& Davidson, B. J. (1980). Attention and the detection of signals. Journal of Experimental Psychology: General, 109, 160-174.

RATCLIFF, R. (1979). Group reaction time distributions and an analysis of distribution statistics. Psychological Bulletin, 86, 446-461.

VAN DER HeIJden, A. H. C., LA HeIJ, W. (1982). The array size function in simple visual search tasks: A comparison between "go- no go" and "yes-no" tasks under conditions of high and low targetnoise similarity. Psychological Research, 44, 355-368.

VAN der Heijden, A. H. C., \& LA HeIJ, W. (1983). The array size function in simple visual search tasks: A comparison between a "gono go" and a "detection" task under conditions of low target-noise similarity. Psychological Research, 45, 221-234.

Van der Heudeen, A. H. C., La Heis, W., \& Boer, J. P. A. (1983). Parallel processing of redundant targets in simple visual search tasks. Psychological Research, 45, 235-254.

\section{NOTES}

1. It is worthwhile to briefly consider the consequences of the assumption that also for the present experiment the intercept measures a distraction decrement. The assumption entails that the values in Columns $\mathrm{Er}$, $\mathrm{El}$, and $\mathrm{A}$ are too large. From the way in which the latencies and percentiles are used in Inequality 2 , Equation 3 , and the fourfold tables needed for the computation of the correlations, it follows directly that (1) the values in Columns O-A and O-B are too low, and a positive value has to be added; and (2) the correlations are too low, and a positive value has to be added (and fourfold tables with both-smaller-than-t entries will tend to disappear). In short, the consequence of the assumption is that the results of the present experiment will move further in the direction of separate activation.

2. It might be proposed that the values for intercept and slope found in our experiment may be related to the relatively brief exposure duration used relative to the exposure durations used by Grice et al. (1984). But, if there is an effect of exposure duration, it is much more likely that this effect will show up in the parameters of the exponential growth functions that enter in the combination rule. There is no a priori or theoretical reason to also expect such an effect to show up in the parameters of the combination rule. Furthermore, if the parameters of the combination rule really depend on exposure duration, Grice et al.'s summation rule loses considerably in generality. In order to assess the generality of the combination rule, however, the issue is worth further investigation.

(Manuscript received March 19, 1984; revision accepted for publication December 6,1984 .) 